\title{
Coalition of distributed generation units to Virtual Power Players - a game theory approach
}

\author{
Hugo Morais, Tiago M. Sousa, Gabriel Santos, Tiago Pinto, Isabel Praça and Zita Vale
}

\begin{abstract}
Smart Grids (SGs) have emerged as the new paradigm for power system operation and management, being designed to include large amounts of distributed energy resources. This new paradigm requires new Energy Resource Management (ERM) methodologies considering different operation strategies and the existence of new management players such as several types of aggregators. This paper proposes a methodology to facilitate the coalition between distributed generation units originating Virtual Power Players (VPP) considering a game theory approach. The proposed approach consists in the analysis of the classifications that were attributed by each VPP to the distributed generation units, as well as in the analysis of the previous established contracts by each player. The proposed classification model is based in fourteen parameters including technical, economical and behavioural ones. Depending of the VPP strategies, size and goals, each parameter has different importance. VPP can also manage other type of energy resources, like storage units, electric vehicles, demand response programs or even parts of the MV and LV distribution network. A case study with twelve VPPs with different characteristics and one hundred and fifty real distributed generation units is included in the paper.
\end{abstract}

Keywords: Decision-making, distributed generation, electricity markets, game theory, intelligent agents coalitions, Virtual Power Players

\section{Introduction}

\subsection{Background and aim}

The increase of distributed generation (DG) and other types of Distributed Energy Resources (DER), such as demand response, storage systems and electric vehicles [35] has brought about new challenges to the electrical network's operation [11], mainly to the distribution system and electricity markets [6]. The high penetration of several types of DER in power systems can be a reality in a near future [2] requiring that all electricity markets and power system players are able to face the new challenges [27] considering an hierarchical and distributed level of decision with a high information dependency level [4].

Despite the favourable scenario for the growth of DER, there are important aspects to consider, both of economic and technical nature [16]. Issues such as the dispatch ability (namely in wind and photovoltaic technologies), the participation of small producers, consumers and storage units in the electricity market increases the need for new approaches and new rules to integrate these resources into a competitive environment context [9].

Virtual Power Players (VPPs) aggregate multiresource, multi-technology and multi-site heterogeneous entities, which can enable overcoming some of 
the most critical problems [18]. One of the main tasks of VPPs is the coalition process of distributed generation units and other energy resources' owners. Coalition process is crucial for the success of the VPP, aiming to establish profitable contracts with low energy acquisition prices. Each VPP can have different coalition goals, considering not only the energy price, but also many other parameters with high relevance for the VPP. During the coalition process, VPPs should consider several aspects such the expectable generation costs, dispatchability, reliability, installed power capacity use, life cycle, volatility, complementary markets, location, GEE emissions, installed power capacity, type of technology, social impact, technology maturity and commercial behaviour.

Several VPPs can actuate in the same region, trying to aggregate the same players (owners of different distributed energy resources) negotiating in the same markets and using the same electric network. This means that a largely competitive environment between VPPs can be expected. The use of game-theory algorithms can be included in decision support tools, providing useful information for the VPPs and for all other players that act in this competitive environment.

The increasing of competition in electricity sector increased significantly in the last years with the sector liberalization [3]. In this competition environment the use of Noncooperative and Cooperative game theory methods can provide decision support solutions in different situations. As example, in [32] a cooperative game theory method is proposed to deal with the sellerto-buyer matching in microgrids perspective. Also to be used in microgrids, in [40] a noncooperative game theory method is proposed to control the energy resources. The approach intents to determine the equilibrium between the generation and the consumption. The method considers the controllable generation and the flexible behaviour in the demand side.

More focus in the electricity market environment, [39] evaluates game-theoretic solution and competitive equilibrium for different kinds of market considering the Nash equilibrium and its refinements for several variants of the architecture for oligopolistic electricity markets. An auction game model for analyzing strategic behaviour in pool-based electricity markets is introduced in $[7,28]$. An incentive mechanism based on the signalling game theory is proposed in [14] and shortterm electricity market auction using game analysis is considered in [34]. Concerning the strategies to wind farms participation in electricity markets a game theory and stochastic programming is proposed in [37]. A vision of next-generation retail electricity market with high penetration of distributed residential electricity suppliers is proposed in [36] considering an innovative game theoretic framework.

All the mentioned approaches uses the game theory to manage the offers (buying/consumption or selling generation). Instead, in this paper, the game theory approach is used to support the agents' coalition. The most important contribution comprises:

- A classification method of distributed generation units to be used by the aggregators. This classification method allows ordering the DG unit by a merit order according the aggregator preferences and strategies. The classification method considers fourteen classification parameters considering technical, economical and behavioural ones. Each aggregator can consider the total or only a part of parameters, assigning different weights for each one;

- The use of a game theory approach for decision support in the coalition negotiation process. Based in the classification process and in the negotiation history, this method allows determining the best negotiation strategy considering different negotiation scenarios.

VPPs and DG are modelled as agents $[8,23]$ in a multi-agent based electricity market simulator called MASCEM (Multi-Agent System for Competitive Electricity Markets) [25,29]. In MASCEM, each market player is a complex and autonomous entity with distinct objectives, purposes and behaviours, taking their own decisions. The complexity of dynamic market players, their interaction and medium/long term gathering of information (data and experience in the market) is therefore modulated in it. In MASCEM, each agent intends to obtain a best results as possible (maximise the incomes to sellers and minimize the outcomes to buyers) considering its technical characteristics and generation/consumption profiles and flexibility. The agents can adapt the strategies according their market performance. To constantly improve its performance and to adapt the strategy to the current environment, each agent includes a learning mechanism [38] to evaluate the success in each negotiation session adjusting the initial strategy if required.

\subsection{Virtual Power Player}

Virtual Power Player can be defined as a market player that aggregates several types of Distributed Energy Resources, namely distributed generation, de- 


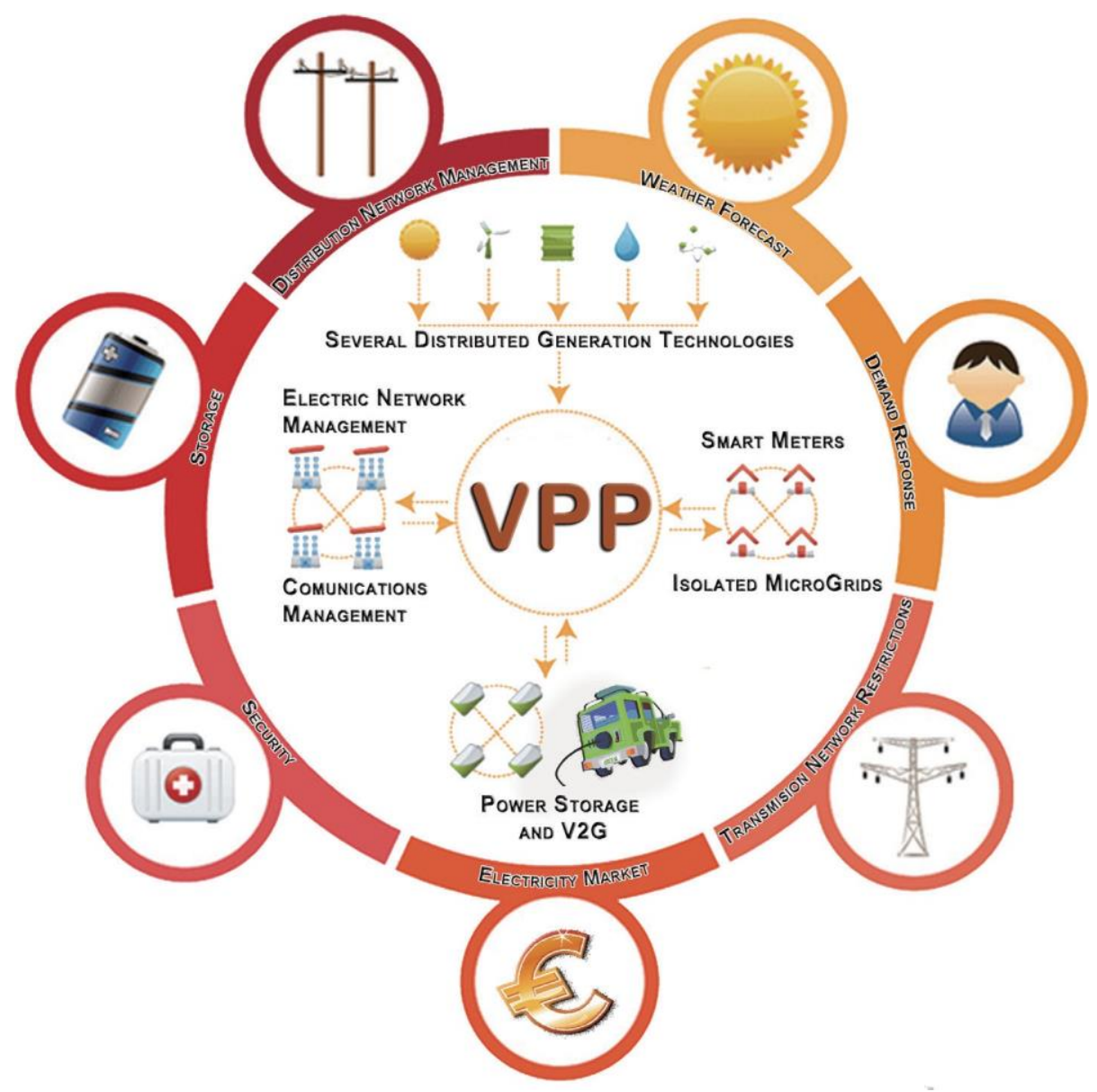

Fig. 1. VPP energy resources management.

mand response, storage units, electric vehicles, load consumption and a part of the distribution network (see Fig. 1) [17,30].

The main task of VPPs is to coordinate the DER operation and to participate in the electricity markets to sell and/or to buy energy according to the aggregated DER availabilities and necessities. In this paper the coalition between VPPs and DG units is addressed considering different VPPs profiles and strategies [26]. DERs have several advantages in being aggregated to a VPP, from which we point out the following ones:

- Market participation - to negotiate the available or necessary energy in the market, an adequate staff is required. If associated to a VPP, the players leave these issues for the VPP to manage. This strategy can also be applied to the other markets, such as carbon markets or district heating. It is still possible to define common strategies for acting in the market and for coordinating the generation with consumer's necessities;
- Maintenance - the maintenance of generation units and storage systems can be ensured by the VPP. The main consequence is the maintenance cost reduction for their owners. If some players have similar technologies, it is possible to create stocks, as well as a single maintenance team for all the players;

- Project - the experience acquired by the VPP will facilitate the new power plants licensing process. The advantages can be reflected at the bureaucratic level and to obtaining credit advantages;

- Forecasting - to ensure a good operation, VPPs need a set of techniques to adequately forecast the consumption, the generation, the electric vehicles mobility [33] and the electricity market price [12];

- Energy Resources Management - the energy resources management will be conducted by the VPP, which will ease the players' operation;

- Participation in demand response (DR) events VPPs should manage the participation of con- 
sumers and electric vehicles in DR events. VPPs can also develop specific DR programs dedicated to aggregated players.

Despite the advantages, players may lose autonomy and consequently prefer to manage their own resources, considering the higher risk associated to this option.

\subsection{Paper structure}

After this introductory section, Section 2 explains the proposed methodology and the coalition process. A case study is presented in Section 3. Finally, Section 4 presents the most relevant conclusions of the paper.

\section{Distributed generation aggregation}

Players' coalition is essential for the VPP creation and evolution, having high implications in all VPP structure and strategies. The negotiation process can be long and complex, therefore a very concrete definition of the objectives to reach is essential $[1,15]$.

In order to improve the negotiation process, each participant entity must ensure an adequate preparation, including the negotiation planning (trying to predict all the possibilities of proposals, and meeting evolution) [24]; the argumentation preparation, in a way that it can be coherent and consistent; and the study of the opposition profile, with the purpose of anticipating its thoughts and actions in different situations. This approach allows the participants to preserve a good relationship and it consists of five basic principles [13]:

- Separating people from the problems;

- Focusing on the interests rather than on positions;

- Creating mutual gain options;

- Persisting on objective (rather than subjective) criteria;

- Developing alternatives for reaching an agreement.

In the first step the VPP should define its global strategy (goals, mission, vision, etc.) and the market segment (aggregation of large-scale renewable based generation units, microgeneration units, CHP, etc.). Considering its global strategy, the VPP will try to define metrics to evaluate the players and posteriorly use these metrics to classify the players in the market that can be aggregated. These processes ensure the people separation from the problems and also the focus on the interests rather than on positions. Based in the play-

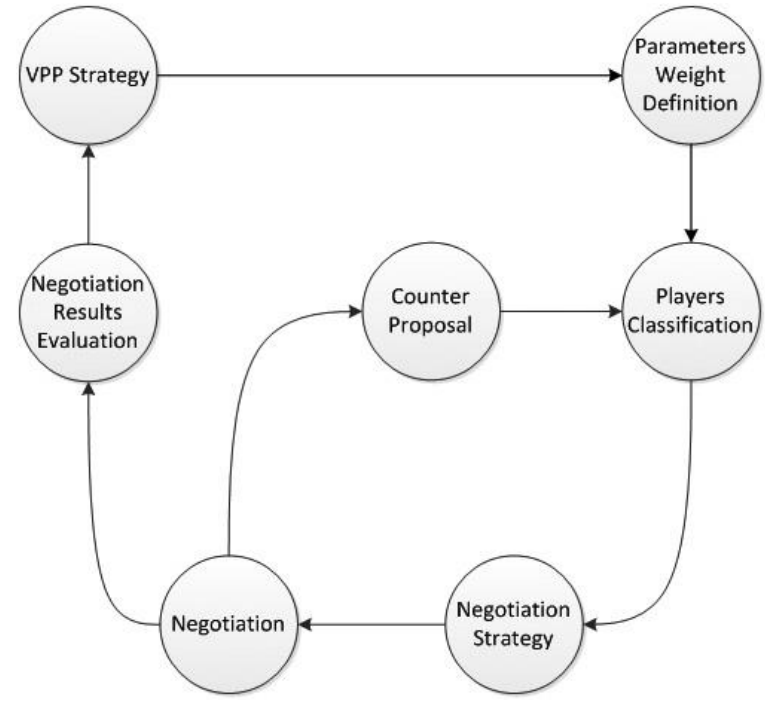

Fig. 2. Negotiation process.

ers' classification, VPPs can define different negotiation strategies for each player. The negotiation is iterative and can include several proposals and counter proposals. VPPs can re-classify the players in each it-

eration considering the new conditions and the new knowledge about the players. Once the negotiation process is concluded, an evaluation of the results should be performed and the global strategies can be adjusted.

In the following sub-sections a description of the negotiation processes between the VPP and distributed generation units are presented.

\subsection{VPP strategy and parameters weight definition}

The strategy of the VPP is partially imposed by the market segment desired by the VPP and by the established financial and trading energy goals [19]. However, the VPP has to define the best strategy to achieve these goals, taking advantage of its market knowledge. This strategy shall define intermediate objectives for the processes, so that its evolution and the accomplishment of the established goals can be monitored. The VPP is able to change the initial strategies, depending on intermediate achieved results [26].

The adopted strategies and the goals to reach must be dynamic and flexible implying a constant analysis of the aggregated players and of the external players that can be interesting for aggregation in future negotiation processes. This evaluation should be continuously performed in order to start negotiations at any time. 
Defining objectives means establishing evaluation parameters for which targets can be attributed. Fourteen evaluation parameters have been considered, for which it is possible to define objectives and weights to be used by VPPs to give more or less importance to each parameter:

- Expectable generation costs $(g c)$ - weight $\left(\psi_{g c}\right)$;

- Dispatchability $(d p) \quad$ - weight $\left(\psi_{d p}\right)$;

- Reliability $(r l) \quad$ - weight $\left(\psi_{r l}\right)$;

- Installed power capacity use $(c u)$ - weight $\left(\psi_{c u}\right)$;

- Life cycle $(l c) \quad$ - weight $\left(\psi_{l c}\right)$;

- Volatility $(v l) \quad$ - weight $\left(\psi_{v l}\right)$;

- Complementary markets $(\mathrm{cm}) \quad$ - weight $\left(\psi_{\mathrm{cm}}\right)$;

- Location $(l t)$

- GHG emissions (ce)

- Power capacity $(p c)$

- Type of technology $(t t)$

- Social impact (si)

- Technology maturity $(\mathrm{tm})$

- Commercial behaviour $(c b)$

- weight $\left(\psi_{l t}\right)$;

- weight $\left(\psi_{c e}\right)$;

- weight $\left(\psi_{p c}\right)$;

- weight $\left(\psi_{t t}\right)$;

- weight $\left(\psi_{s i}\right)$;

- weight $\left(\psi_{t m}\right)$;

- weight $\left(\psi_{c b}\right)$.

Each VPP must assign a relative importance to each parameter, and define a specific weight for each one, allowing the players' classification. Depending of their goals and their market segment, VPPs can be classified in different types [25]. In the present approach six VPP types are considered, with the following characteristics:

- Parallel VPP (PVPP) - It includes different producers with distinct generation capacities, typically larger than $1 \mathrm{MW}$ and lower than $20 \mathrm{MW}$. The common characteristic of this type of VPPs is their participation in parallel markets (district heating, $\mathrm{CO} 2$, etc.);

- Large Scale VPP (LSVPP) - It includes producers with large generation capacity, typically higher than $10 \mathrm{MW}$;

- Global VPP (GVPP) - This type of VPP can aggregate all types of distributed energy resources, including the distributed generation, consumers with demand response contracts, storage systems including electric vehicles. Other important aspect on this type of VPP is the capability of managing part of the distribution network;

- Integrated VPP (IVPP) - This type of VPP is similar to the GVPP. However, the IVPP does not include the distribution network management capability. The IVPP can aggregate large scale energy resources;

- Several VPP (SVPP) - This type of VPP does not have a priori defined characteristics, aggregating different types of energy resources with different energy capacities;

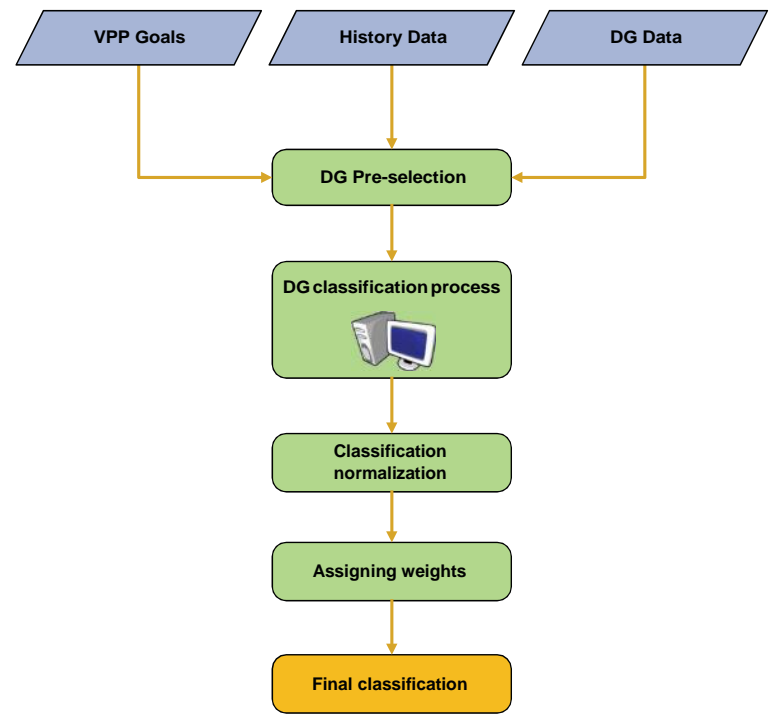

Fig. 3. Classification flowchart for generation units.

- Micro VPP ( $\mu$ VPP) - This type of VPP is composed of many producers with small capacity, typically lower than $2 \mathrm{MW}$.

\subsection{Players classification}

A player's classification process was designed allowing the evaluation of each producer. Figure 3 presents the classification process flowchart.

The classification process uses the VPP's goals to evaluate each producer. The knowledge about each player is different and VPPs use some history data to better characterize the player. For example, the VPP can assume the installed capacity use of a photovoltaic unit considering the installed capacity use of similar units in the same region. The information regarding the players can be improved during the negotiation process.

Based in the VPP goals and in the producer characteristics, the VPP must execute a players' preselection. Based on a small set of criteria it can immediately exclude the possibility of coalition. For example, specifying a players' minimum or maximum dimension, or imposing limits in $\mathrm{CO} 2$ and other GHG emissions.

After the pre-selection, VPPs evaluate the producers in each classification parameter described in Section 3.1. In order to become possible to compare classifications assigned by VPPs, it is necessary to normalize the obtained values. In this approach, all classification values are normalized in a scale from -5 to 5 , in 
which the negative values are assigned to players with parameters outside the range of values that was defined as acceptable for the VPP.

After the normalization process each parameter is multiplied by the weight that the VPP assigned to it. The final classification is achieved through a weighted evaluation of all parameters, according to Eq. (1).

$$
\begin{aligned}
& \text { Class }_{V P P \rightarrow \text { Prod }}= \\
& g c \times \psi_{g c}+d p \times \psi_{d p}+r l \times \psi_{r l}+c u \times \psi_{c u} \\
& +l c \times \psi_{l c}+v l \times \psi_{v l}+c m \times \psi_{c m} \\
& +l t \times \psi_{l t}+c e \times \psi_{c e}+p c \times \psi_{p c}+t t \times \psi_{t t} \\
& +s i \times \psi_{s i}+t m \times \psi_{t m}+c b \times \psi_{c b}
\end{aligned}
$$

In the classification process, VPPs must always consider the impact that each player is expected to have in the VPP's structure, i.e., the VPP must bond the current value of the parameter under evaluation with the value it would reach if the player is aggregated to the coalition. In cases when the VPP is starting its operation, there are no data available from its previous operation. Therefore it should use the values defined as objectives to execute the classification process, as well as the expected values for each parameter of each player.

\subsection{Negotiation strategy}

VPPs can have different strategies in the negotiation process using some decision support tools in order to identify the best one in different negotiation scenarios. VPPs compete with each other in order to obtain advantages in the negotiation process. The use of a game theory based approach can be very important for the VPPs negotiation in order to define the best strategy considering different negotiation scenarios.

Game theory can be applied in cases of interaction between players (people or entities) mutually aware that the individual result depends on and influences the actions of the others [5]. The first approaches concerning game theory have emerged in [20], having gained an increased importance after the publication of this concept in [21]. Currently, game theory is used in a diversity of areas, such as math, economy, sociology, biology, political science, psychology, computer science, among other $[10,22,41]$.

The use of game theory in the coalition process consists in the analysis of the classifications that were attributed by each VPP to the distributed generation units, as well as in the analysis of the previous established contracts by each player. The analysis of the previous contracts that have been established by each player with other VPPs provides a strong indicator of

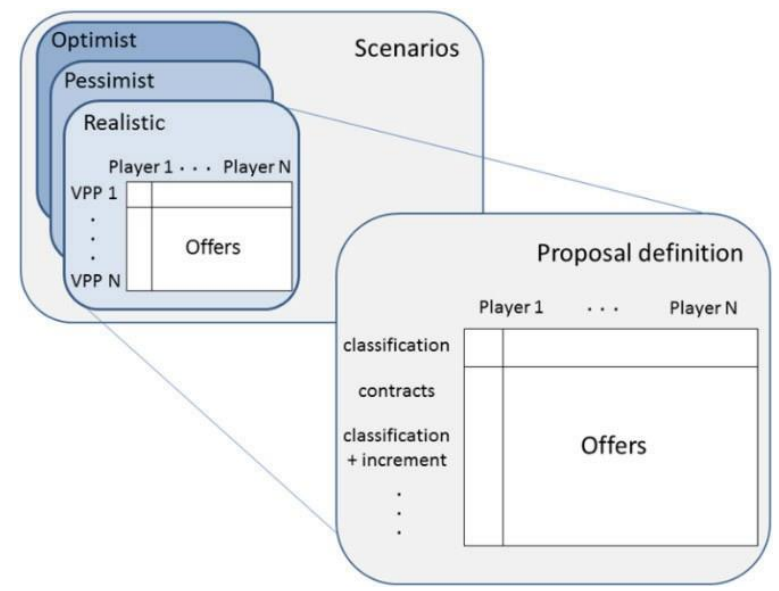

Fig. 4. Scenarios and proposals matrices.

the deals that can be expected to be achieved when negotiating with each of these players. This evaluation is used to create a matrix with the history of the proposed offers, which is used in the coalition offers development algorithm. Each VPP can establish a different parameter's weight for the analysis of the contracts and for the classifications (2). As the history of previous contract information Cont prev is most of the times incomplete, VPPs will tend to assign higher weights to the classifications value Class $V P P \rightarrow$ Prod. However the weights can be adjusted during the negotiation process considering the improvement of the knowledge regarding the players' behaviour.

$$
\begin{aligned}
\text { Offer }_{G T}= & \text { Class }_{V P P \rightarrow \text { Prod }} \times \vartheta_{\text {Class }} \\
& + \text { Cont }_{\text {prev }} \times \vartheta_{\text {Cont }}
\end{aligned}
$$

VPPs define matrices considering different VPPs offers to each player. Matrices allow the best negotiation strategy definition in each scenario. The developed algorithm considers three base scenarios (Fig. 4). The realistic scenario assumes that the other VPPs will maintain their behaviour, i.e. assuming they will use the same strategy of past negotiations. In the implemented method, each VPP can determine the importance value of each player for the other VPPs considering the same classification method but changing the parameters weights according to the knowledge about the other VPPs. The optimist scenario considers that the VPPs will place offers with values that are more favourable for the VPPs. The pessimist scenario considers proposals with higher advantages for the generation units.

After defining the distinct scenarios, new matrices are created, representing the possible actions that a 


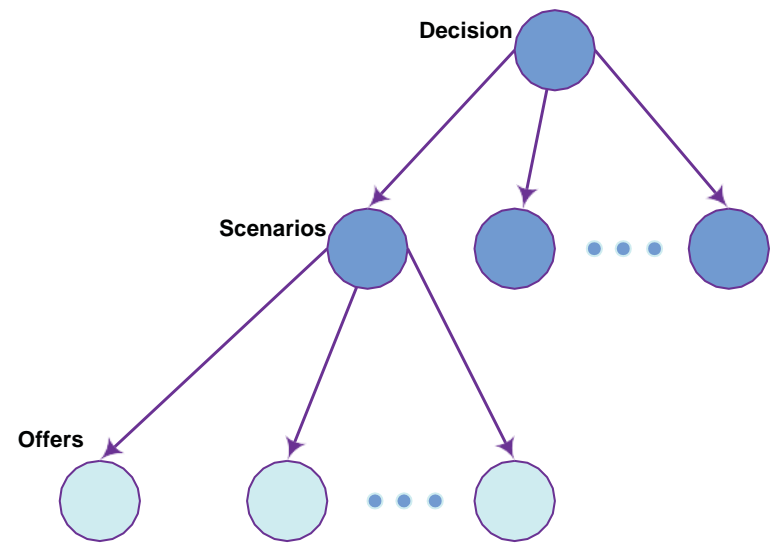

Fig. 5. Scenarios analysis process.

VPP can adopt. Each line of these matrices symbolizes the offers that a VPP presents to each generation unit. The offers are based on the reference values obtained from the combination of the players' classification, and the analysis of the history data of previous contracts, if available. Since a wide range of alternative offers is required, several possible offers are generated. The resulting offers are tested against each of the considered scenarios.

Each set of offers is represented by the nodes of the lower level, i.e., the "Offers" of the tree represented in Fig. 5. The intermediate nodes represent a defined scenario, each one with a set of offers. Each of these analysis "Scenarios" is conducted through a simulation, which results in a utility value.

The utility value for each VPP $\left(U_{V P P}\right)$ is characterized by the total amount of power that a VPP is able to achieve from the coalitions, as the sum of the power that is contracted with each producer $\left(P_{P r o d}\right)$; and it is penalized proportionally to the weighted average of payments agreed with each generation unit (Pay Prod $)$. This way, the utility value will be higher as the amount of achieved power gets closer to the amount that the VPP desires, and which contracts with players present the lower costs for the VPP.

$$
\begin{aligned}
U_{V P P} & =\frac{P_{\text {Prod }}}{\frac{\left(\text { Pay }_{\text {Prod }} \times P_{\text {Prod }}\right)}{P_{\text {Prod }}}} \\
& =\frac{\left(P_{\text {Prod }}\right.}{\left(\text { Pay }_{\text {Prod }} \times P_{\text {Prod }}\right)}
\end{aligned}
$$

Depending on the evaluation values for each set of offers in the considered scenarios, each VPP can adjust its strategy to suit its acting profile and its objectives and long term strategy. For that, a decision method is used with the purpose of determining which set of actions, or final offers, will be, in fact, presented as proposals to the players. Figure 6 presents the parameters used in the proposals definition.

The proposed methodology considers six decision methods, which correspond to six different VPP acting profiles. The considered profiles comprise:

- The realistic method is used as the reference case. The values proposed by the VPPs are simply based on the classifications assigned by each VPP to each player, so that these results can be compared to the other decision methods, and hence conclusions can be taken about the advantages of the use of each method;

- The pessimist method represents the typical case of a cautious VPP, i.e., the usual minimax method application. In this case, the chosen scenario is the one in which the worst results were achieved (the worst scenario a VPP can find - mini), and the chosen set of offers is the one that got the best results for this scenario ( $\max$ );

- On the contrary, the optimist method will opt for the scenario with the highest simulation advantages, being chosen the best set of offers for this scenario;

- The probable method was implemented to deal with the variation of the probability of being able to account for a credible history of contracts with the players. Therefore, this method allows that when this history is not available, or the information able to be retrieved from that data is not sufficient, only the scenarios based on the classifications are considered;

- The tight method considers for the scenario analysis only the proposals that are equal or inferior to the reference values obtained from the history analysis of classifications and contracts;

- Finally, the gluttonous method considers only the scenarios, from which the simulation results are most satisfactory, i.e., for which the proposals are able to originate the best results for the VPP.

The proposals generated by the decision method are the offers that are effectively considered for the negotiation with players, from the VPP whose actions are being supported by the decision method based on game theory. Figure 6 - Scenarios and proposals definition in game theory algorithm 


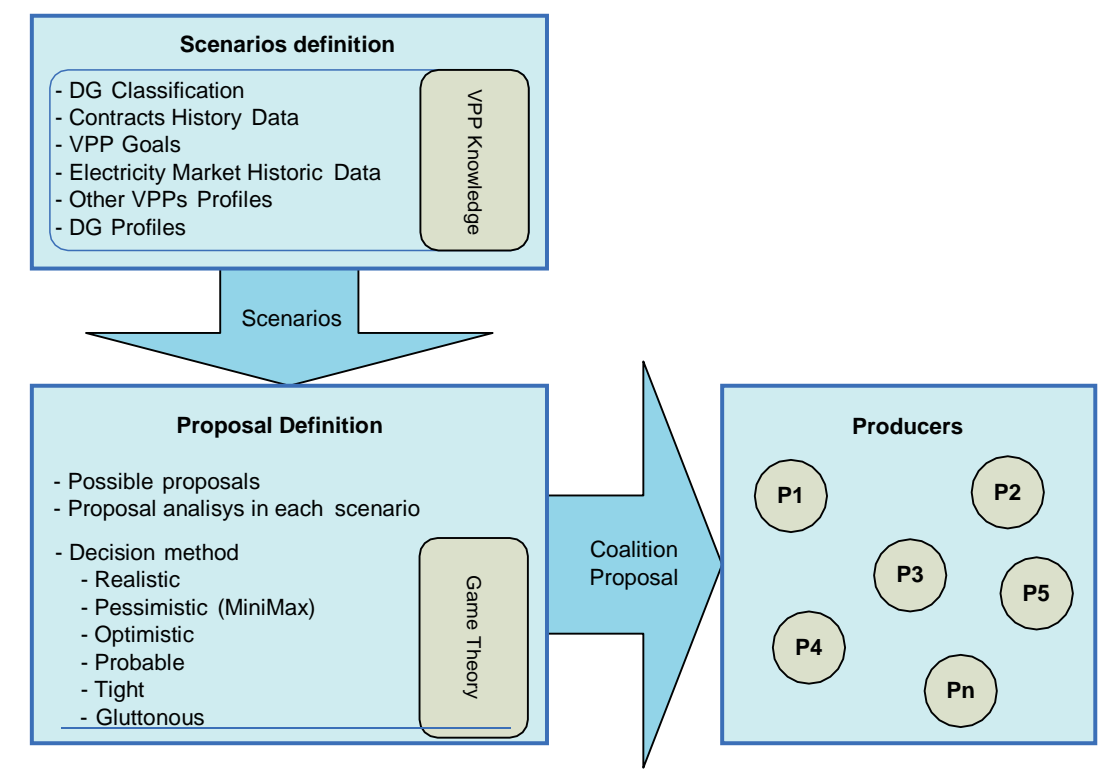

Fig. 6. Scenarios s and proposals definition in game theory algorithm.

\section{Case study}

This case study intends to show the application of the classification and negotiation mechanisms presented in this paper. In order to make this case study as realistic as possible, 150 real generation units were selected from a data base which includes approximately 40.000 units from Spain [31]. The producers were selected considering the average capacity of power units of each technology and the number of units of each technology in a specific region of Spain. Additionally, 12 VPPs with multiple characteristics and objectives are considered. The number of VPPs was defined in order to understand the developed processes, considering a competition between players but also different goals and market segments position.

The real available characteristics of generation units are the technology, the installed power capacity, the location, and the year of entry into service. The other classification elements were established according to the expected values for each generation technology. All information regarding the producers' characteristics can be consulted in www.gecad.isep.ipp.pt/IES. Figure 7 presents the classification results.

The simulation of the negotiation process between VPPs and players, using the proposed game theory approach, uses the classifications presented in Fig. 7, and a history of previous contracts signed by each player. In opposite side to the classification process, which was based on real data from the generation units, the history of previous contracts has been built manually, since there is no available data to be used regarding this aspect.

In order to test the proposed methodology, several scenarios have been built considering the use of game theory model by all VPPs, and also the use by only one VPP. Regarding the scenarios for which all VPPs use game theory approach, four possibilities have been tested:

- All VPPs adopt the Realistic strategy (based on the classification of the players), with weight of 1 for the classification, and weight of 0 for the history of previous contracts;

- All VPPs adopt the Realistic approach, with weight of 0.5 for both the classification and the history of previous contracts;

- All VPPs adopt the Probable strategy, with weight of 1 for the classification and weight of 0 for the history of previous contracts;

- All VPPs adopt the Probable approach, with weight of 0.5 for both the classification and the history of previous contracts.

Table 1 presents the VPPs aggregations results by technology using game theory for all VPPs considering a Realistic strategy and the weight 1 to the players' classification. The numbers in the table represent the aggregated players. The column of power $(\mathrm{P})$ shows the aggregated capacity and inside the parenthesis the capacity goals for each VPP. Comparing the aggre- 


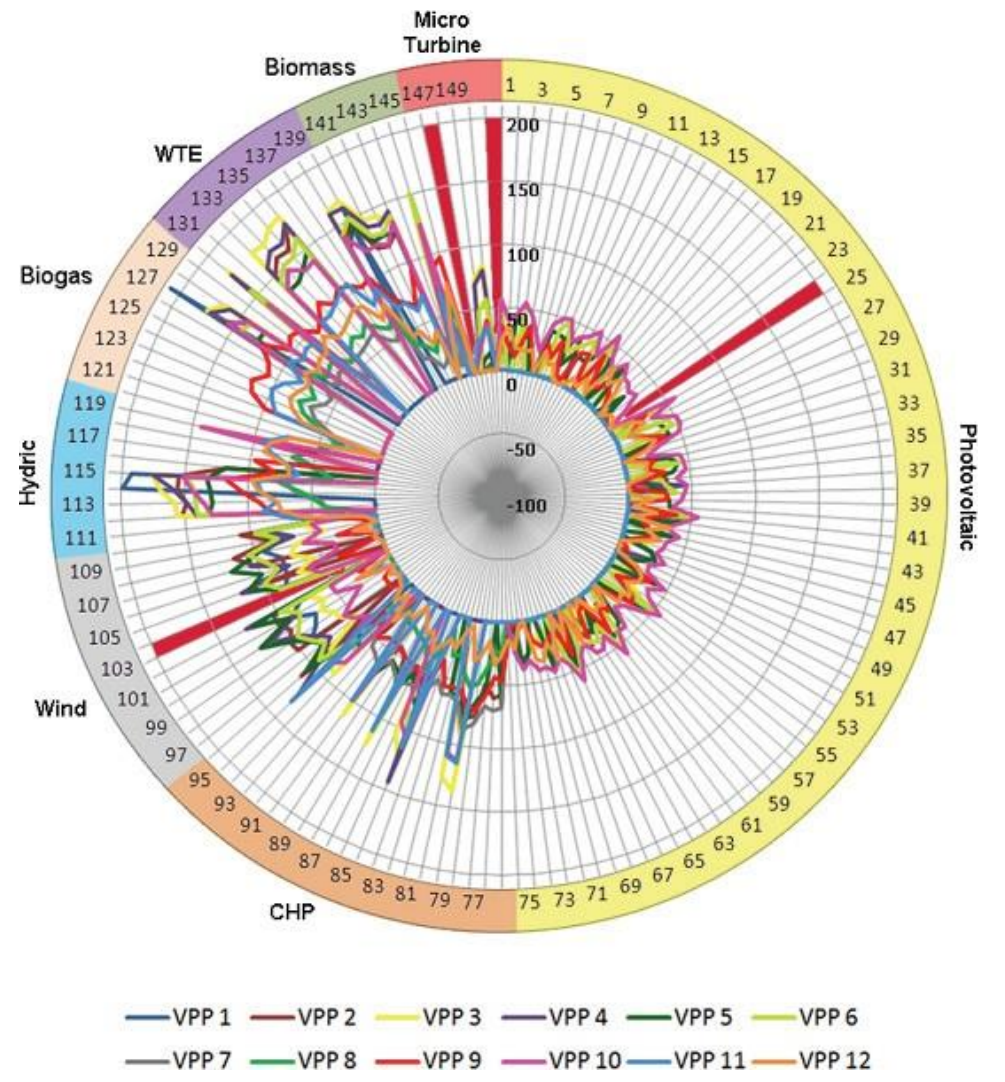

Fig. 7. Classification attributed by the VPPs the production units.

gated power capacity and the VPPs goals it is possible evaluate the success in the negotiation process.

Figure 8 presents the aggregations results using game theory for all VPPs considering different scenarios. Since the VPPs present very different scales, it was necessary to divide the figure into two in order to easily show the variations instigated by the change in parameters.

From Fig. 8 it is possible to see that using the Realistic strategy, even by changing the weight for classifications and contracts, the results are kept unchanged. However, when using the Probable profile, significant changes are verified in the coalition results. VPP 1 and VPP 10 are the most penalized ones, as in this context they were not able to aggregate any generation unit. Analysing the obtained results in Realistic strategy, and taking into consideration that not all VPPs compete against each other, it is visible that the VPPs that got the worst results were VPP 6 and VPP 12 (VPPs with less aggregated capacity when compared with the required ones). VPP 6 is a LSVPP, which competes directly with the other LSVPPs, with the IGVPP, and in some cases with SVPPs and PVPPs. These VPPs try to aggregate players with high installed power capacity. VPP 12 is a $\mu$ VPP and competes with VPP 10 and VPP 11, and not so directly with VPP 1 (GVPP), VPP 7 (SVPP), and VPPs 8 and 9, which are of the type PVPP.

Taking these results into consideration, we decided to apply the proposed game theory methodology to the two VPPs that got the worst results (VPP 6 and VPP 12), to understand if their results can be improved using this method. Each one of the two VPPs will be tested with different acting profiles (Realistic, Pessimistic, Optimistic, Probable, Tight, and Gluttonous). Figure 9 shows the results obtained for VPP 6, and Fig. 10 shows the results obtained for VPP12.

In Fig. 9 it is possible to see the impact in aggregation results of game theory use only by VPP6. It is also important to refer that, excluding the Gluttonous decision strategy, which originates the non-aggregation of any player. All other strategies originate notoriously better results for VPP 6 when compared to the probable and realistic methods (results inFig. 8).

From Fig. 10 it is possible to see the change in the decision method used by VPP 12 only interferes with 
Table 1

Coalitions results for each VPP using the classification method

\begin{tabular}{|c|c|c|c|c|c|c|c|c|c|}
\hline VPP & Photov. & $\mathrm{CHP}$ & Wind & Small hydro & Biogas & WTE & Biom. & Micro turbine & $\overline{\mathrm{P}}(\mathrm{MW})$ \\
\hline 1 & - & - & - & $114 ; 115$ & 127 & - & & - & $1.12(1)$ \\
\hline 2 & - & - & $\begin{array}{c}96 ; 98 ; \\
99 ; 101 ; \\
108 ; 110\end{array}$ & 116 & - & - & - & - & 125 (148) \\
\hline \multirow[t]{2}{*}{3} & - & 80; 81; & & $112 ; 113$ & $128 ; 130$ & $\begin{array}{c}132-135 \\
138-140\end{array}$ & 141 & $148 ; 149$ & $159(200)$ \\
\hline & & $\begin{array}{l}86 ; 88 ; \\
91 ; 94\end{array}$ & & & & & & & \\
\hline 4 & - & 85 & $102 ; 105$ & - & - & - & 142 & - & $105(500)$ \\
\hline 5 & - & - & $\begin{array}{l}97 ; 100 ; \\
104 ; 106 ; \\
107 ; 109\end{array}$ & - & - & - & - & - & $182(500)$ \\
\hline 6 & - & - & - & - & - & - & 144 & - & $21(200)$ \\
\hline 7 & - & $\begin{array}{c}76-79 ; \\
82-84 ; \\
87 ; 89 ; \\
90 ; 92 ; \\
93\end{array}$ & - & - & - & - & 143 & - & $16.2(25)$ \\
\hline 8 & - & - & - & - & 121 & - & - & - & $1.3(20)$ \\
\hline 9 & - & - & - & 117 & $\begin{array}{c}122-126 \\
129\end{array}$ & $131 ; 137$ & 145 & - & $15.2(15)$ \\
\hline 10 & $\begin{array}{c}1-17 \\
19-23 \\
25-67 \\
69-74\end{array}$ & - & - & - & - & - & - & - & $0.76(1)$ \\
\hline 11 & - & 95 & - & $111 ; 120$ & - & 136 & - & - & 0.63 (1) \\
\hline 12 & $\begin{array}{c}18 ; 43 ; \\
68 ; 75\end{array}$ & - & - & 118 & - & - & - & - & 0.37 (1) \\
\hline N.A. & - & - & 103 & 119 & - & - & - & $\begin{array}{c}146 ; 147 \\
150\end{array}$ & 5.9 \\
\hline
\end{tabular}

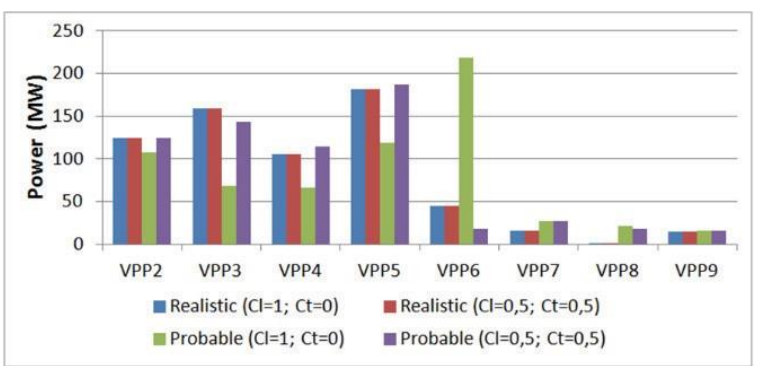

(a)

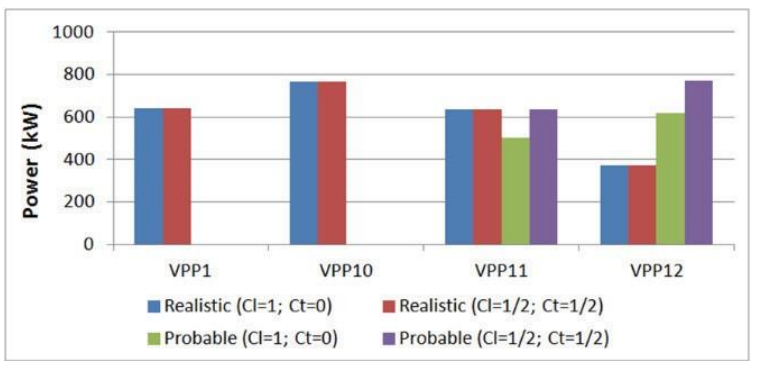

(b)

Fig. 8. Results of the coalition process with all VPPs using game theory approach for: (a) VPPs of types IVPP, LSVPP, SVPP and PVPP; (b) VPPs of types GVPP and $\mu$ VPP.

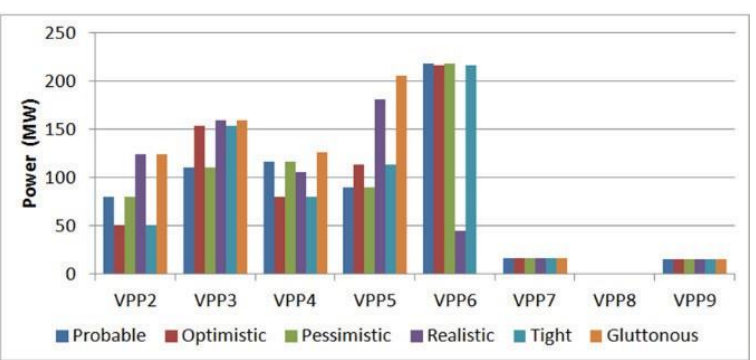

(a)

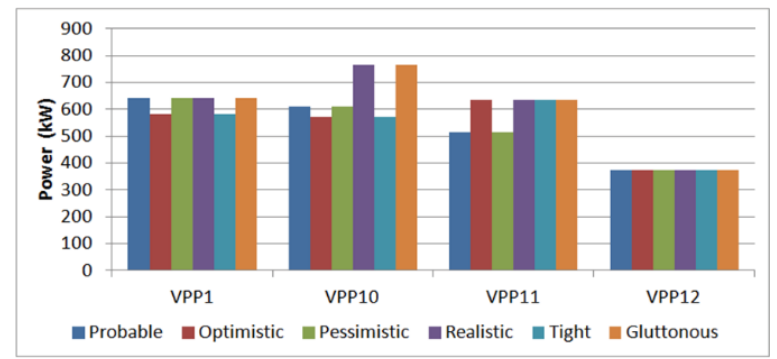

(b)

Fig. 9. Results of the coalition process with VPP 6 using game theory approach for: (a) VPPs of types IVPP, LSVPP, SVPP and PVPP; (b) VPPs of types GVPP and $\mu$ VPP. 


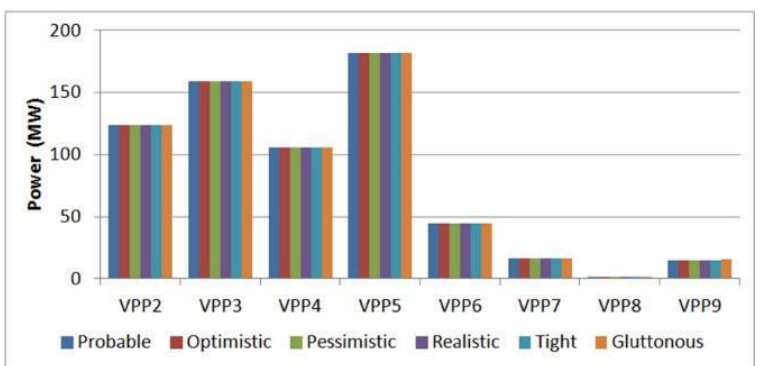

(a)

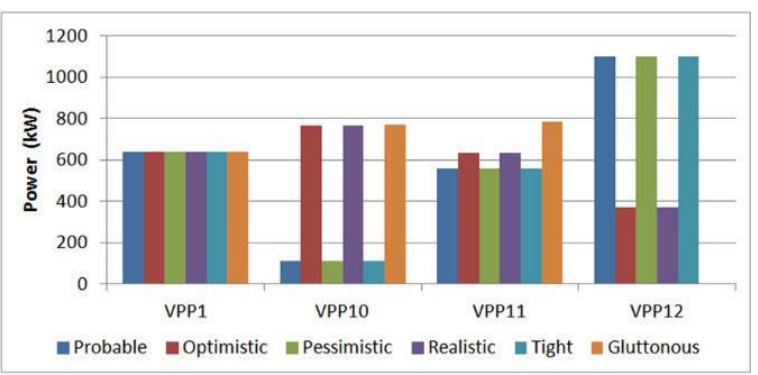

(b)

Fig. 10. Results of the coalition process with VPP 12 using the game theory approach for: (a) VPPs of types IVPP, LSVPP, SVPP and PVPP; (b) VPPs of types GVPP and $\mu$ VPP.

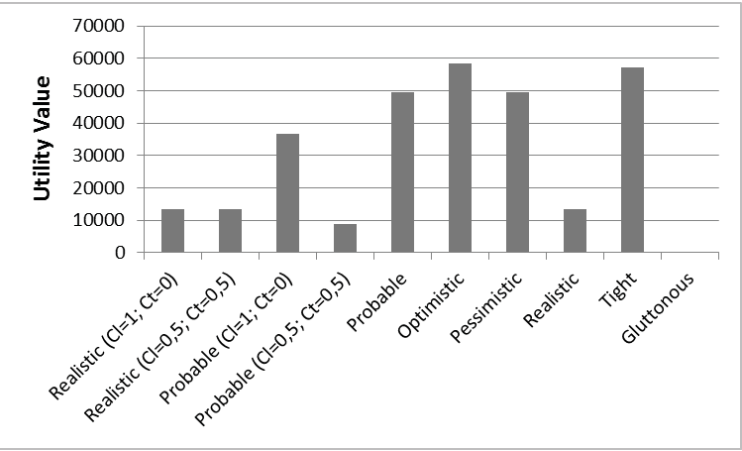

Fig. 11. Utility values of VPP 6 in each of the considered scenarios.

the aggregation of VPPs of type $\mu \mathrm{VPP}$, which are direct competitors of VPP 12. Once again, the Gluttonous decision method originates the aggregation of no players, while all the other decision methods have shown a huge improvement in VPP 12 results.

Figure 11 presents the comparison of the utility values (UVPP) that evaluate the performance of VPP 6 in each of the scenarios. UVPP, as shown in Eq. (3), presents higher values when the total volume of aggregated power increases, and as the paid price for this power decreases; i.e. UVPP potentiates the achievement of large amounts of aggregated power at the lower possible contracted prices.

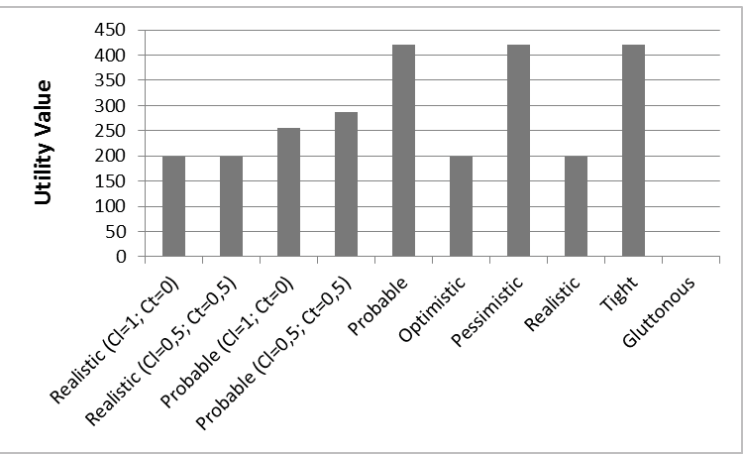

Fig. 12. Utility values of VPP 12 in each of the considered scenarios.

From Fig. 11 it is possible to see that, comparing to the four reference cases (in Fig. 8), which are represented by the first four bars of the graph of Fig. 11, the game theory methods have presented much higher utility values. With the exception of the Gluttonous method, which resulted in no aggregations, and the Realistic method, which achieved an utility value equal to the first two reference methods; all other game theory based methods have originated better results for VPP6. This supports the conclusion that, not only the game theory methods provide larger aggregated power amounts, but also at lower prices. The best example is the third reference scenario (third bar from the graph Probable $(\mathrm{Cl}=1 ; \mathrm{Ct}=0))$, which has originated a similar amount of aggregated power for VPP 6 (as can be seen from Fig. 8) when compared to the four game theory methods that originated the best results (see Fig. 9). However, even though gathering the same amount of power, the achieved utility value is much lower, which means that the same amount of power was aggregated at the cost of much higher payments.

Figure 12 presents the utility value comparison for VPP 12 in all the considered scenarios.

Figure 12 shows that, from the six game theory methods, three were able to nearly double the utility value of the reference cases. Two others achieved similar utility values to those of the reference cases, and the Gluttonous method achieved a null value to its inability to gather any aggregated power. An important conclusion to be taken from Fig. 12 is that, besides the fact that the game theory methods were able to potentiate the results of VPP12, the methods that were able to achieve the best results were the Probable, Pessimistic, and Tight methods. Comparing to the results of VPP6, one can see that these three methods were able to achieve good results for both VPPs. However, in the case of VPP 6 (Fig. 11) the method that achieved the best results from all (even better than these three) 
was the Optimistic method. The Optimistic method was not able to maintain the good results for VPP 12 . From this, the conclusion to be taken is that the Probable, Pessimistic, and Tight methods are the most reliable ones, and show to be capable of achieving very good results regardless of the supported player. The Optimistic method, on the other hand, is the method that achieved the best results for VPP 6 , but it did not perform as well for VPP 12; i.e. this method is a riskier approach, which is able to achieve the best results from all methods, but it does not offer guarantees of success.

Considering the obtained results, the VPPs, in this case the VPP6 and VPP12, should analyse what is the best strategy to adopt in real negotiation, trying to identify the most probable real negotiation scenarios and make the offers based in the game theory results.

\section{Conclusions}

The negotiation between VPPs and generation units is crucial for the success of VPPs' operation. The more advantageous the coalition contracts are, the most competitive the VPP can become. However, it is important to preserve the interests of the entities that own the generation units, because VPPs operate in a competitive market, in which players can aggregate to other VPPs, or, in some cases, participate directly in electricity markets, without necessarily requiring the coalition with a VPP.

In this paper it has been proposed the use of the game theory approach for decision support in the coalition negotiation process between VPP and distributed generation producers, considering all the required con-

ditions and factors. The developed proposed method allows VPPs to adjust their strategies according to different scenarios and according to the other VPPs' profiles.

With the objective of simulating the coalition process in a competitive environment, a scenario based on real Spanish generation units was built. In this scenario, IVPPs and LSVPPs compete to aggregate generation units with high installed power capacity, while GVPPs and $\mu$ VPPs try to attract lower dimension players. SVPPs and PVPPs essentially competed for the medium dimension players, but they also had some influence on small and large players' aggregation.

As shown by the results of the presented case study, the use of the proposed game theory approach has clear implications in the coalition results of VPPs 6 and 12, which were not able to get good results without the use of this method for supporting their decisions. Once the proposed method was applied to these VPPs, the quality of their results clearly increased. Different strategic profiles and decision methods led to different results, some of them more advantageous than others, yet all perfectly applicable and notoriously advantageous for the decision support in the coalition formation process.

\section{Acknowledgments}

This work is supported by FEDER Funds through COMPETE program and by National Funds through FCT under the projects FCOMP-01-0124-FEDER: PEst-OE/EEI/UI0760/2011, PTDC/SEN-ENR/122174 12010.

Hugo Morais is funding from the The Danish Council for Strategic Research under grant agreement no. 11-116794 (SOSPOProject).

\section{References}

[1] S. Adhau, M.L. Mittal and A. Mittal, A multi-agent system for distributed multi-project scheduling: An auction-based negotiation approach, Engineering Applications of Artificial Intelligence 25(8) (2012), 1738-1751.

[2] R. Badawy, A. Yassine, A. Heßler, B. Hirsch and S. Albayrak, A novel multi-agent system utilizing quantum-inspired evolution for demand side management in the future smart grid, Integrated Computer-Aided Engineering 20(2) (2013), 127141.

[3] S. Ceppi and N. Gatti, An algorithmic game theory study of wholesale electricity markets based on central auction, Integrated Computer Aided Engineering 17 (2010), 273-290.

[4] S. Distefano and A. Puliafito, Information dependability in distributed systems: The dependable distributed storage system, Integrated Computer-Aided Engineering 21(1) (2014), 3-18.

[5] W. Elsner, T. Heinrich and H. Schwardt, The Microeconomics of complex economies, The microeconomics of Complex Economies, Elsevier, 2015, pp. 25-32.

[6] H. Farhangi, The path of the smart grid, IEEE Power and Energy Magazine 8(1) (2010), 18-28.

[7] D. Gan, J. Wang and D. Bourcier, An auction game model for pool-based electricity markets, International Journal of Electrical Power Energy Systems 27(7) (2005), 480-487.

[8] J.O. Gutierrez-Garcia and K.M. Sim, Agent-based cloud workflow execution, in: Integrated Computer-Aided Engineering 19 (2012), 39-56.

[9] A.D. Hawkes and M.A. Leach, Modelling high level system design and unit commitment for a microgrid, AppliedEnergy 86(7) (2009), 1253-1265.

[10] J.C. Hennet and Y. Arda, Supply chain coordination: A gametheory approach, Engineering Applications of Artificial Intelligence 21(3) (2008), 399-405.

[11] L. Jia, Y. Wang and L. Fan, Multiobjective bilevel optimization for production-distribution planning problems using hybrid genetic algorithm, Integrated Computer-Aided Engineering 21(1) (2014), 77-90. 
[12] D. Li, L. Xu, E.D. Goodman, Y. Xu and Y. Wu, Integrating a statistical background- foreground extraction algorithm and SVM classifier for pedestrian detection and tracking, Integrated Computer-Aided Engineering 20(3) (2013), 201-216.

[13] B. List and B. Korherr, An evaluation of conceptual business process modelling languages, in: Proceedings of the 2006 ACM Symposium on Applied Computing - SAC '06, New York, New York, USA: ACM Press, (2006), p. 1532.

[14] Z. Liu, X. Zhang and J. Lieu, Design of the incentive mechanism in electricity auction market based on the signaling game theory, Energy 35(4) (2010), 1813-1819.

[15] A.T. Marty, Getting to YES, Negotiating agreement without giving in, Critical Care Medicine, 1983.

[16] B.V. Mathiesen, H. Lund and K. Karlsson, 100\% renewable energy systems, climate mitigation and economic growth, $A p$ plied Energy (2011).

[17] H. Morais, P. Kádár, P. Faria, Z. Vale and H.M. Khodr, Optimal scheduling of a renewable micro-grid in an isolated load area using mixed-integer linear programming, Renewable Energy 35(1) (2010), 151-156.

[18] H. Morais, T. Pinto, Z. Vale and I. Praca, Multilevel negotiation in smart grids for VPP management of distributed resources, IEEE Intelligent Systems 27(6) (2012), 8-16.

[19] K. Nagy and K. Körmendi, Use of renewable energy sources in light of the "New Energy Strategy for Europe 2011-2020", Applied Energy 96 (2012), 393-399.

[20] J. Neumann, Zur theorie der gesellschaftsspiele, Mathematische Annalen 100(1) (1928), 295-320.

[21] J.V. Neumann and O. Morgenstern, Theory of games and economic behavior, 1944 .

[22] A. Osborne and J. Martin, An introduction to game theory, 2004.

[23] E. Ostrosi, A.J. Fougeres, M. Ferney and D. Klein, A fuzzy configuration multi-agent approach for product family modelling in conceptual design, Journal of Intelligent Manufacturing 23 (2012), 2565-2586.

[24] S. Park and S.B. Yang, An efficient multilateral negotiation system for pervasive computing environments, Engineering Applications of Artificial Intelligence 21(4) (2008), 633-643.

[25] T. Pinto, H. Morais, P. Oliveira, Z. Vale, I. Praça and C. Ramos, A new approach for multi-agent coalition formation and management in the scope of electricity markets, Energy Elsevier(2011).

[26] T. Pinto, I. Praca, Z. Vale, H. Morais and T. Sousa, Strategic bidding in electricity markets: An agent-based simulator with game theory for scenario analysis, Integrated ComputerAided Engineering (2013).
[27] T. Pinto, Z. Vale, T.M. Sousa, I. Praça, G. Santos and H. Morais, Adaptive learning in agents behaviour: A framework for electricity markets simulation, Integrated Computer-Aided Engineering 21(4) (2014),399-415.

[28] M. Prabavathi and R. Gnanadass, Energy bidding strategies for restructured electricity market, International Journal of Electrical Power Energy Systems 64 (2015), 956-966.

[29] I. Praca, C. Ramos, Z. Vale and M. Cordeiro, MASCEM: A multiagent system that simulates competitive electricity markets, IEEE Intelligent Systems 18 (2003).

[30] D. Pudjianto, C. Ramsay and G. Strbac, Virtual power plant and system integration of distributed energy resources, IET Renewable Power Generation 1(1)(2007), 10.

[31] REE, Red Elétrica de España, Retrieved from www.ree.es, 2013.

[32] W. Saad, Z. Han and H.V. Poor, Coalitional game theory for cooperative micro-Grid distribution networks, in: 2011 IEEE International Conference on Communications Workshops (ICC), IEEE (2011), 1-5.

[33] J. Sedano, C. Chira, J.R. Villar and E.M. Ambel, An intelligent route management system for electric vehicle charging, Integrated Computer-Aided Engineering 20 (2013), 321-333.

[34] Y.S. Son, R. Baldick, K.H. Lee and S. Siddiqi, Short-term electricity market auction game analysis: Uniform and payas-Bid pricing, IEEE Transactions on Power Systems 19(4) (2004), 1990-1998.

[35] T. Sousa, J. Soares, Z. Vale and H. Morais, Day-ahead resource scheduling in smart grids considering vehicle-to-Grid and network constraints, Applied Energy (2012).

[36] W. Su and A.Q. Huang, A game theoretic framework for a next-generation retail electricity market with high penetration of distributed residential electricity suppliers, Applied Energy 119 (2014), 341-350.

[37] D. Ting and Q. Wei, Trading wind power in a competitive electricity market using stochastic programing and game theory, IEEE Transactions on Sustainable Energy 4(3) (2013), $805-815$.

[38] Z. Vale, T. Pinto, I. Praca and H. Morais, MASCEM: Electricity markets simulation with strategic agents, IEEE Intelligent Systems 26(2) (2011),9-17.

[39] Vasin, Game-theoretic study of electricity market mechanisms, Procedia Computer Science 31 (2014), 124-132.

[40] W.W. Weaver and P.T. Krein, Game-theoretic control of small-scale power systems, IEEE Transactions on Power Delivery 24(3) (2009), 1560-1567.

[41] M. Wooldridge, The triumph of rationality, IEEE Intelligent Systems 27(1) (2012), 60-64. doi:10.1109/MIS.2012.20. 